\title{
Ionic force field optimization based on single-ion and ion-pair solvation properties: Going beyond standard mixing rules
}

\author{
Maria Fyta ${ }^{1,2}$ and Roland R. Netz ${ }^{1,3}$ \\ ${ }^{1}$ Physik Department, Technische Universität München, 85748 Garching, Germany \\ ${ }^{2}$ Institut für Computerphysik, Universität Stuttgart, 70569 Stuttgart, Germany \\ ${ }^{3}$ Fachbereich Physik, Freie Universität Berlin, 14195 Berlin, Germany
}

(Received 23 September 2011; accepted 23 February 2012; published online 22 March 2012)

\begin{abstract}
Using molecular dynamics (MD) simulations in conjunction with the SPC/E water model, we optimize ionic force-field parameters for seven different halide and alkali ions, considering a total of eight ion-pairs. Our strategy is based on simultaneous optimizing single-ion and ion-pair properties, i.e., we first fix ion-water parameters based on single-ion solvation free energies, and in a second step determine the cation-anion interaction parameters (traditionally given by mixing or combination rules) based on the Kirkwood-Buff theory without modification of the ion-water interaction parameters. In doing so, we have introduced scaling factors for the cation-anion Lennard-Jones (LJ) interaction that quantify deviations from the standard mixing rules. For the rather size-symmetric salt solutions involving bromide and chloride ions, the standard mixing rules work fine. On the other hand, for the iodide and fluoride solutions, corresponding to the largest and smallest anion considered in this work, a rescaling of the mixing rules was necessary. For iodide, the experimental activities suggest more tightly bound ion pairing than given by the standard mixing rules, which is achieved in simulations by reducing the scaling factor of the cation-anion LJ energy. For fluoride, the situation is different and the simulations show too large attraction between fluoride and cations when compared with experimental data. For NaF, the situation can be rectified by increasing the cation-anion LJ energy. For $\mathrm{KF}$, it proves necessary to increase the effective cation-anion Lennard-Jones diameter. The optimization strategy outlined in this work can be easily adapted to different kinds of ions. () 2012 American Institute of Physics. [http://dx.doi.org/10.1063/1.3693330]
\end{abstract}

\section{INTRODUCTION}

The faithful molecular modelling of ionic effects in a biomolecular context typically requires explicit-water computer simulations using effective, classical interactions, known as force fields. A set of standard and well-tested force fields for water exist. ${ }^{1,2}$ Such explicit water models can be quite simple, involving only two ${ }^{3}$ or three interaction sites corresponding to the atoms of water, such as TIPS, ${ }^{4} \mathrm{SPC},{ }^{5}$ TIP3P, ${ }^{1}$ and SPC/E. ${ }^{6}$ There also exist more complicated water models, which include dummy atoms, such as the various 4-, 5-, and 6-site models. ${ }^{1,7-14}$ The former, simpler water models are less accurate, but computationally less demanding than the latter ones, and can describe many of the relevant water properties surprisingly well. The development of reliable ionic force fields is essential for applications involving ionic solutions in biology, technology, and physical chemistry ${ }^{15-17}$ and has been a field of interest for many years. As an example in the context of biomolecules, the ion specificity in the preference of binding to biomolecular surfaces ${ }^{18}$ or on nucleic acids ${ }^{19}$ and the molecular mechanisms involved are of high importance towards a deeper understanding of biological processes, as well as in view of biotechnological applications. Classical atomistic simulations are not as accurate as quantum mechanical approaches. However, they are computationally much less demanding and thus allow to treat larger spatial and temporal scales, which is essential for many problems involving the distribution and exchange of ions. In combination with explicit water models that exhibit realistic dielectric properties, classical ion models describe the electrostatic interactions between ions and charged groups typically quite robustly, without the need to make additional assumptions on the type and strength of solvent mediated forces. ${ }^{20,21}$ The accuracy of all obtained results, though, depends on the precise form of the force field. ${ }^{22}$ Many simulation approaches to ionic solutions use classical pairwise additive non-polarizable potentials. These potentials are often argued to implicitly take into account polarizability effects, which are intrinsically of many-body nature. ${ }^{23}$ This is corroborated by works that have shown that the first hydration shell of mono- and divalent ions is only weakly polarized (except orientational effects), ${ }^{24}$ thereby accounting for polarizability effects seems not to be strictly required for ions. Nevertheless, it is clear that a force field with more parameters would generally be expected to be more accurate if properly optimized with respect to all of its parameters. Another important issue is related to force-field transferability. Often, force fields are parameterized with respect to experimental data in the crystalline state, ${ }^{25,26}$ and it is a priori not clear how such force fields will perform in the solvated aqueous state.

Different approaches have been followed to optimize ionic force fields. In the first studies, the properties of single ions, such as ionic solvation free energies or ionic hydration structures in small water clusters, were used for the parameterization of force fields. ${ }^{27}$ Since a Lennard-Jones (LJ) potential involves two parameters, typically expressed as the 
LJ interaction diameter and the LJ energy, a single experimental observable does not determine the force field uniquely. Recently, two studies have revisited single-ion optimization strategies, systematically explored the two-dimensional LJ force-field parameter space and attempted to optimize two experimental observables simultaneously. ${ }^{28,29}$ It turned out, though, that depending on the observable chosen (free energy of solvation, entropy of solvation, or radius of solvation) the cationic parameters could not be optimized in a fully satisfactory fashion. ${ }^{29}$ It has also been demonstrated that monovalent ion parameters can lead to physically inaccurate behavior in molecular dynamics simulations of strong electrolytes already at concentrations well below the solubility limit. A fix has been proposed, which is generalizable for simulating diffuse and specific ion binding to nucleic acids. ${ }^{30}$ Another common approach for optimizing ionic parameters through alteration of the mixing rules is the so-called NBFIX modification used in the CHARMM molecular dynamics program. ${ }^{31}$ It is based on a solvent boundary potential approach and reproduces free energies of solvation for the solutions. This approach has proven efficient in studies on the conduction and ion selectivity through potassium channels. ${ }^{32,33}$ Note that for low concentrations as the ones considered here, the calculated thermodynamics properties based on the LorentzBerthelot (LB) mixing rules match with those from the NBFIX method. ${ }^{34}$ As was also shown very early, force-field optimization in the dilute limit often fails to reproduce realistically the structure and thermodynamics of the electrolytes at non-vanishing concentrations, even for simple ionic solutions such as $\mathrm{NaCl}^{35-37}$ This is not surprising, since at elevated salt concentration the cation-anion interactions become important and dominate the solution thermodynamics. Weerasinghe and Smith used Kirkwood-Buff integrals (KBIs) as determined by experimental activities for $\mathrm{NaCl}$ solutions in order to optimize the force fields. ${ }^{35}$ In similar applications of Kirkwood-Buff solution theory (KBT) (Ref. 38) mixtures of various cosolutes and ions with water ${ }^{39-43}$ or the cation specific binding onto protein surface charges were treated. ${ }^{44}$ The SPC water model was used together with the Kirkwood-Buff-derived force field ${ }^{35}$ for the development of various alkali force fields. ${ }^{45} \mathrm{In}$ a recent work, KBT-derived force fields for the whole suite of alkali halides were reported. ${ }^{23}$ Thermodynamic integration is an alternative method to calculate the thermodynamic properties of solutions and has been used for the calculation of osmotic coefficients and hydration free energies. ${ }^{34,46,47} \mathrm{In}$ this work we restrict ourselves to the KBT approach.

This summary of previous works might give the erroneous impression that when optimizing ionic force fields, a choice has to be made whether to accurately model finiteconcentration behavior using KBT (benchmarked with respect to experimental activity coefficients, compressibilities, and partial volumes), or to reproduce the infinite-solution limit based on single-ion solvation parameters and effective ionic radii. In fact, in a recent paper we demonstrated an alternative approach where we attempted to simultaneously satisfy both single-ion and ion-pair (i.e., finite concentration) properties. ${ }^{48}$ In the two-dimensional LJ parameter space, we first determined parameter values for the ion-water LJ interactions that reproduce the experimental free energy of solvation.
These parameter combinations turn out to correspond to continuous lines in the two-dimensional space spanned by the LJ diameter and LJ energy. ${ }^{29}$ In a second step, we confined the search onto these lines and determined solvation properties at finite ion concentration. In that work, we used the osmotic pressure as the optimized property, and determined ion parameters that faithfully reproduce the experimental osmotic coefficients at a given finite ion concentration for a few representative halide-alkali ion pairs. The resulting force fields therefore satisfied single-ion and ion-pair properties at the same time. That study showed, however, that a full optimization was not possible for all ion pairs. Especially ion pairs including fluoride and iodide ions were problematic.

In the present work, we reconsider the problem of generating ionic force fields based on simultaneous optimizing single-ion and ion-pair properties. As single-ion property we again choose the free energy of solvation, thereby connecting to our earlier work ${ }^{29,48}$ and the historic optimization strategies. ${ }^{27,49-53}$ In contrast to our earlier work, ${ }^{48}$ here we use KBT and optimize simulation results with respect to experimental activities, similar to the other KBT-derived force fields. Our approach, however, differs fundamentally from previous works in that we (i) fix ion-water parameters based on single-ion optimization, and (ii) that we determine the cation-anion interaction parameters (normally given by mixing or combination rules) based on KBT without modification of the ion-water interaction parameters. This seems like a natural strategy, since the bare water-ion interactions are not expected to change due to the presence of other ions (note that ion-water distribution functions of course do change with salt concentration, but these effects are caused by multibody correlation effects included in any statistical mechanics treatment of solutions). We strictly stay on the pair-potential level, i.e., the potential function can be decomposed into pairpotentials, and we choose as target for optimization in this work the cation-anion LJ interaction. We argue that before enlarging the parameter space of the interaction model and consider more complicated force fields (for example by including atomic polarizabilities), one should rather explore the already existing force-field parameter space in all possible depth. No solid foundation for the combination rules that are used in common atomistic MD simulations seems to exist; since the cation-anion interaction becomes important at non-zero salt concentration, this interaction seems to be a natural target for optimization. In most situations, we modify the overall prefactor of the cation-anion LJ interaction, the LJ energy, similar to previous studies which modify the LJ energy through the cation-water interaction. ${ }^{23,35,44}$ For KF, which constitutes a particularly problematic ion pair, we also modify the cationanion LJ diameter that appears in the KF-LJ interaction. We show that with this modification of the ionic force fields, experimental thermodynamic properties both at infinitely dilute and finite concentrations can be reproduced. We also discuss possible reasons for the complications encountered when trying to model ion-pairing for different ions and show different scenarios of how cation-anion distribution functions reflect the crossover from direct-ion pairing to solvent-separated ion pairing. The paper is organized as follows: in Sec. II, we present the methodology used in this study and the choice 
of the force-field parameter space, in Sec. III we discuss the results and the calculated KBIs, and we briefly conclude in Sec. IV.

\section{METHODS}

\section{A. Simulation details}

We perform atomistic simulations using the MD package GROMACS (Refs. 54 and 55) in the $(N, P, T)$ ensemble, with fixed particle number $N$, pressure $P=1$ bar, and temperature $T=300 \mathrm{~K}$. The Berendsen barostat and thermostat are used. $^{56}$ The simulation box is cubic, with an edge length of $L$ $=4 \mathrm{~nm}$, and is periodically repeated in all three dimension. The initial configurations of the salt solutions are generated from a cubic box of equilibrated water molecules by randomly replacing water by ions until the desired ion concentration is reached. We typically use a total number of 2156 SPC/E water molecules. ${ }^{6}$ Within this water model, oxygen and hydrogen atoms are connected by rigid bonds and carry partial charges optimized in such a way that important water properties (density, structure, surface tension, dielectric constant) are well reproduced. The system is large enough so that finite size effects are not significant, ${ }^{57}$ as explicitly checked by the convergence of the KB integrals. The three dimensional particlemesh Ewald sum was used for the electrostatic interactions ${ }^{58}$ with a grid spacing in Fourier space of $0.12 \mathrm{~nm}^{-1}$ in all three directions. We have used an interpolation order of 4 , a distance cutoff of $0.9 \mathrm{~nm}$ for the real-space interactions, and a relative strength of the electrostatic interaction at the cutoff of $10^{-5}$. No long-range correction for the cutoff dispersion has been used. Typical times for the simulations for gathering statistics are 100-150 ns. These simulation parameters have been shown to be efficient for modelling ionic solutions and to yield well-converged properties (most importantly radial distribution functions (RDFs)) both at low and high salt concentrations. $^{48,57}$

\section{B. Mixing rules and parameter space}

Eight different salt solutions are modeled in this study as representative test cases, namely $\mathrm{NaCl}, \mathrm{NaF}, \mathrm{NaBr}, \mathrm{NaI}$, $\mathrm{KCl}, \mathrm{KF}, \mathrm{CsCl}$, and $\mathrm{CsI}$ at an electrolyte density of $0.31 \mathrm{~m}$ corresponding to 12 anions and 12 cations in the solution. The molality $0.31 \mathrm{~m}$ corresponds to a Molarity of about $0.3 \mathrm{M}$. Ions were modeled as non-polarizable spheres interacting via pairwise additive 6-12 $\mathrm{LJ}$ potentials of the form

$$
V_{\mathrm{LJ}}\left(r_{i j}\right)=4 \varepsilon_{i j}\left[\left(\frac{\sigma_{i j}}{r_{i j}}\right)^{12}-\left(\frac{\sigma_{i j}}{r_{i j}}\right)^{6}\right],
$$

with two free parameters, the $\mathrm{LJ}$ diameter $\sigma_{i j}$ and the $\mathrm{LJ}$ en$\operatorname{ergy} \varepsilon_{i j}$, for each pair of atoms. For the SPC/E water model used here, the LJ parameters are $\varepsilon_{O O}=0.6500 \mathrm{~kJ} / \mathrm{mol}$ and $\sigma_{O O}=0.3169 \mathrm{~nm}$ and assigned to the oxygen (the two hydrogen atoms do not interact via $\mathrm{LJ}$ potentials). Partial charges of $q_{O}=-0.8476 e$ and $q_{H}=+0.4238 e$ are assigned to the oxygen and hydrogen atoms of the water molecule, respectively. The whole set $\left\{\sigma_{i j}, \varepsilon_{i j}, q_{i}\right\}$ with $i, j=1, \ldots, M$, in Eq. (1) defines the total force field determining the nonbonded inter- and intra-molecular dynamics interactions for $\mathrm{M}$ atomic species. Typically, the vast number of parameters is reduced by using heuristic mixing rules for the cross interactions $(i \neq j)$ so that the only remaining parameters are the diagonal coefficients $\sigma_{i i}$ and $\varepsilon_{i i}$. The common mixing rules are $\varepsilon_{i j}=\sqrt{\varepsilon_{i i} \varepsilon_{j j}}$, and either $\sigma_{i j}=\left(\sigma_{i i}+\sigma_{j j}\right) / 2$, constituting the LB mixing rules, or $\sigma_{i j}=\sqrt{\sigma_{i i} \sigma_{j j}}$, defining the geometric mixing rules. ${ }^{59}$

In our recent study of aqueous salt solutions, we used the Lorentz-Berthelot mixing rules and could reproduce experimental osmotic coefficients for most ion pairs by adjusting the diagonal ionic force-field parameters $\sigma_{i i}$ and $\varepsilon_{i i}$ constrained on the line that matches the experimental ionic solvation free energies. ${ }^{48}$ However, for some of the salts, such as KF and CsI, this procedure did not work. In the present work, we reconsider halide-alkali ion pairs, and specifically concentrate on the previous problematic ion pairs KF and CsI. As in our previous study, ${ }^{48}$ we mostly consider force-field parameters that lie on the free energy of solvation curve for single ions, which also happen to reproduce the ionic radius with acceptable accuracy. ${ }^{29}$ Along that line, we choose force fields that could reproduce or best approximate the osmotic coefficient, in the case of $\mathrm{Cs}^{+}$and $\mathrm{I}^{-}$we test two different sets of force fields. For sodium $\left(\mathrm{Na}^{+}\right)$, chlorine $\left(\mathrm{Cl}^{-}\right)$, and bromine $\left(\mathrm{Br}^{-}\right)$ we used literature parameters, namely those proposed by Dang, ${ }^{51,52}$ which give reasonable hydration free energies. ${ }^{28,29}$ The $\mathrm{Na}^{+}$force field closely reproduces the hydration free energy curve, while those for $\mathrm{Cl}^{-}$and $\mathrm{Br}^{-}$slightly deviate from the hydration free energy curves. ${ }^{29}$ All Lennard-Jones ionic parameters used in this work are summarized in Table I. For consistency, the numbers in parentheses for some of the ions follow the notation in our previous work. ${ }^{48}$ In this table also the parameters $\varepsilon_{i O}$ and $\sigma_{i O}$ for the ion-water interaction from the Lorentz-Berthelot mixing rules are given. For the

TABLE I. Ion-water and bare ionic parameters for the anions and cations used in the current $\mathrm{MD}$ simulations. $\mathrm{The}$ parameters for $\mathrm{Na}^{+}, \mathrm{Cl}^{-}$, and $\mathrm{Br}^{-}$are taken from Refs. 51 and 52, while the rest is taken from Ref. 48. The Pauling radii of the ions are also given. ${ }^{60}$

\begin{tabular}{|c|c|c|c|c|c|c|c|c|c|}
\hline & $\mathrm{Na}^{+}$ & $\mathrm{Cl}^{-}$ & $\mathrm{K}(11)^{+}$ & $\mathrm{Cs}(6)^{+}$ & $\operatorname{Cs}(9)^{+}$ & $\mathrm{F}(5)^{-}$ & $\mathrm{I}(1)^{-}$ & $\mathrm{I}(4)^{-}$ & $\mathrm{Br}^{-}$ \\
\hline$\sigma_{i O}(\mathrm{~nm})$ & 0.2876 & 0.3785 & 0.293 & 0.333 & 0.325 & 0.3665 & 0.45 & 0.425 & 0.39 \\
\hline$\varepsilon_{i O}(\mathrm{~kJ} / \mathrm{mol})$ & 0.5216 & 0.5216 & 1.26 & 0.5 & 1.0 & 0.1 & 0.1 & 0.32 & 0.52 \\
\hline$\sigma_{i i}(\mathrm{~nm})$ & 0.2583 & 0.440 & 0.269 & 0.3491 & 0.3331 & 0.4161 & 0.5831 & 0.5331 & 0.4631 \\
\hline$\varepsilon_{i i}(\mathrm{~kJ} / \mathrm{mol})$ & 0.4186 & 0.4186 & 2.44 & 0.325 & 1.54 & 0.015 & 0.015 & 0.157 & 0.412 \\
\hline Pauling radius (nm) & 0.095 & 0.18 & 0.133 & 0.169 & 0.169 & 0.124 & 0.225 & 0.225 & 0.198 \\
\hline
\end{tabular}


cation-anion interaction we have introduced scaling factors $\lambda_{\varepsilon}$ and $\lambda_{\sigma}$ for the LJ parameters according to $\varepsilon_{+-}=\lambda_{\varepsilon} \sqrt{\varepsilon_{++} \varepsilon_{--}}$ and $\sigma_{+-}=\lambda_{\sigma}\left(\sigma_{++}+\sigma_{--}\right) / 2$, which describe deviations from the usual Lorentz-Berthelot mixing rule and are in the range $[0.4,3.0]$. Note, that these additional scaling factors leave the ion-water interaction parameters unchanged, in contrast to previous KBT modelling studies. ${ }^{23,35,44}$ Our choice is justified by the robust determination of ion-water interaction parameters from solvation properties, i.e., single-ion properties.

The procedure we follow for the optimization of ionic force fields is the following: for each ion pair, we first vary the scaling factor $\lambda_{\varepsilon}$ and obtain the relevant structural details, namely the radial distribution function of the solution. We then calculate the KBIs for the solution, from which we obtain the derivative of the activity, as will be discussed next. The optimization of the LJ parameter for each ion pair with respect to $\lambda_{\varepsilon}$ is achieved by comparing the calculated activity derivatives with the respective experimental data. This procedure works for all ion pairs except KF. Here, we additionally vary the cation-anion LJ diameter via the scaling factor $\lambda_{\sigma}$, which is shown to work well for this problematic case.

\section{Structural analysis}

A good indicator of electrolyte structural properties are the RDFs, $g_{i j}(r ; \rho)$, which are directly obtained from the MD simulations for a density $\rho$. The RDFs are expected to reach unity at long distances $r$, but due to finite size effects, this is not necessarily the case. ${ }^{61}$ To overcome this, we introduce a correcting factor, so that the corrected RDF used in the calculations of the KBIs is written as $g_{i j}(r ; \rho)=f(\rho) g_{i j}^{\operatorname{sim}}(r ; \rho)$, where $g_{i j}^{\text {sim }}(r ; \rho)$ the RDF obtained directly from the MD simulations and $f(\rho)$ a prefactor of the order of $1 \pm 1 / N$.

\section{Kirkwood-Buff theory of solutions}

The KB theory links solution thermodynamics to distribution functions ${ }^{38}$ via the KB integrals. For two species $i$ and $j$, the $\mathrm{KB}$ integrals have the form:

$$
G_{i j}=\int_{0}^{\infty} 4 \pi r^{2}\left[g_{i j}(r)-1\right] d r .
$$

In this equation $\mathrm{g}_{i j}(r)$ is the RDF (or pair correlation function) between $i$ and $j$ at a distance $r$. For an electrolyte solution, the system is handled as a binary system of water (w) and cosolvent (c). ${ }^{39,62}$ The relevant KB integrals are $G_{w w}$, $G_{c c}$, and $G_{c w}=G_{w c}$. Given the number density $\rho_{j}=N_{j} / V$ of species $j$, with $V$ the system volume, the excess coordination numbers follow as $N_{i j}=\rho_{j} G_{i j}$ and measure the excess or deficit of species around a particle in the actual solution compared to a homogenous solution. A value $N_{i j}<0$ corresponds to depletion of species $j$ in the vicinity of species $i$, while a value of $N_{i j}>0$ denotes an excess. One can also define the partial molar volumes of the salt solution components, as follows:

$$
\begin{aligned}
\bar{V}_{c} & =\frac{1+\rho_{w}\left(G_{w w}-G_{c w}\right)}{\eta}, \\
\bar{V}_{w} & =\frac{1+\rho_{c}\left(G_{c c}-G_{c w}\right)}{\eta},
\end{aligned}
$$

where $\eta=\rho_{w}+\rho_{c}+\rho_{w} \rho_{c}\left(G_{c c}+G_{w w}-2 G_{c w}\right)$. The partial volumes obey the equation $\rho_{c} \bar{V}_{c}+\rho_{w} \bar{V}_{w}=1$.

The KBIs are linked directly to the activity derivatives $\left(\mathrm{a}_{c c}\right)$ and molar activity coefficients $\left(y_{c}\right)$ of a solution. This is obvious from the following relations, for a two component system at constant temperature $T$ and pressure $P$ :

$$
\begin{gathered}
\mathrm{a}_{c c}=\frac{1}{1+\rho_{c}\left(G_{c c}-G_{c w}\right)}=\frac{1}{1+N_{c c}-\left(\rho_{c} / \rho_{w}\right) N_{c w}}, \\
\mathrm{a}_{c c}=\left.\left(\frac{\partial \ln \mathrm{a}_{c}}{\partial \ln \rho_{c}}\right)\right|_{P, T}=1+\left.\left(\frac{\partial \ln y_{c}}{\partial \ln \rho_{c}}\right)\right|_{P, T} .
\end{gathered}
$$

The electrolyte activity $\mathrm{a}_{c}$ is defined through $\mathrm{a}_{c}$ $=y_{c} \rho_{c}$, and is experimentally accessible. The cosolvent number density is $\rho_{c}=N_{c} / V$. The cosolvent-water and cosolvent-cosolvent components of the KBIs that enter the calculation of the activity derivatives (Eq. (4a)) are given by

$$
\begin{gathered}
G_{c w}=\left(G_{w+}+G_{w-}\right), \\
G_{c c}=\frac{1}{4}\left(2 G_{+-}+G_{++}+G_{--}\right) .
\end{gathered}
$$

Additional details on the KBT for ionic solutions can be found elsewhere. ${ }^{35,39,62}$

\section{RESULTS AND DISCUSSION}

\section{A. Structural properties and Kirkwood-Buff integrals}

We first look at the RDFs for each salt and different scaling factors $\lambda_{\varepsilon}$. As a representative example we show the RDFs for CsI in Fig. 1(a), where parameters for Cs9 and I1 in Table I are used. As mentioned before, these force-field parameters were found to be optimal in our previous work. ${ }^{48}$ Small values of $\lambda_{\varepsilon}$ lead to clustering of the ions, evident in the RDFs as very high peaks. The peak positions do not differ significantly among the different $\lambda_{\varepsilon}$ values. One discerns noticeable differences in all three cation-anion, cationcation, and anion-anion RDFs (labels '+-', '++', and '--' in Fig. 1(a), respectively). Variations also in the water-cation and water-anion (labels ' $w+$ ' and ' $w-$ ' in Fig. 1(a), respectively) RDFs are seen. Evidently, for small $\lambda_{\varepsilon}$ prefactors, the ionic clustering also affects the water structure around the ions and the first hydration shells. Similar behavior is also seen for the other ion pairs (data shown in Ref. 63). KBIs are shown in Fig. 1(b) for the case of a Cs9I1 salt solution. All different KBIs are shown, namely cation-anion, cationcation, anion-anion, water-cation, and water-anion. As expected, the specific differences observed in the RDFs among different $\lambda_{\varepsilon}$ values are transferred to the KBIs. In Fig. 1(c) we show the KBI contributions, $G_{c c}$ and $G_{c w}$, that enter the calculation of the activity derivative in Eq. (4a) and that are defined in Eq. (5), for Cs9I1. As a general remark, $G_{c c}$, the 


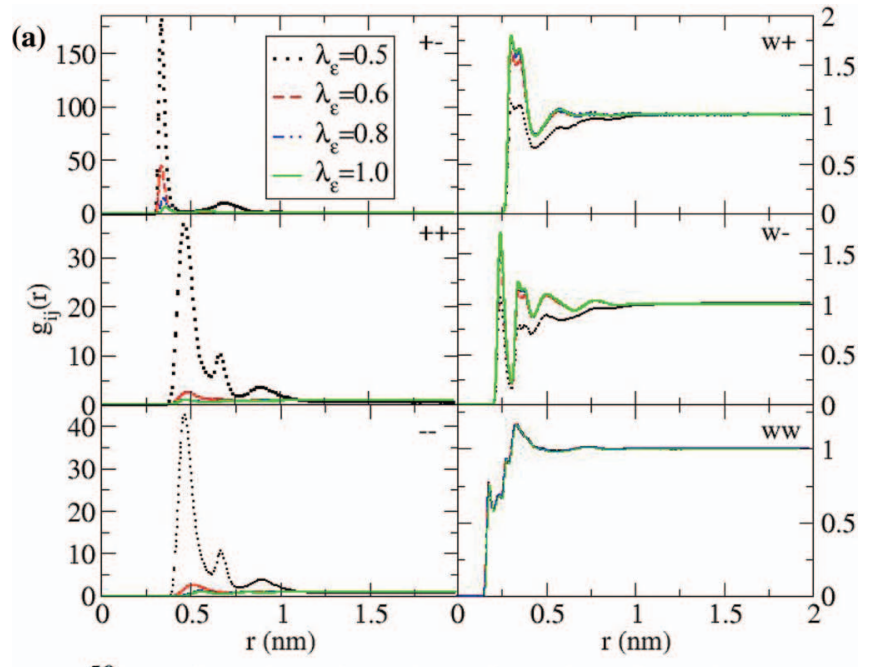

(b)

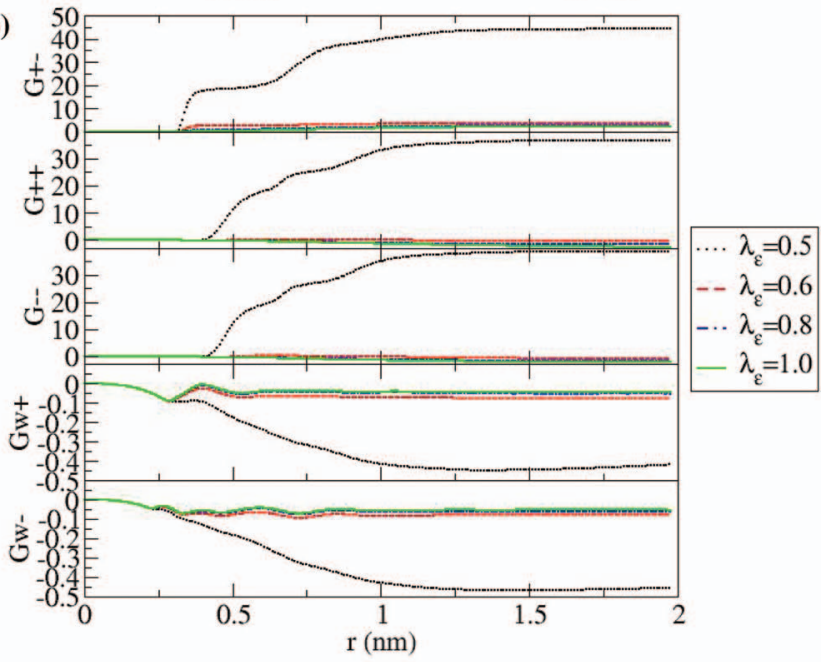

(c)

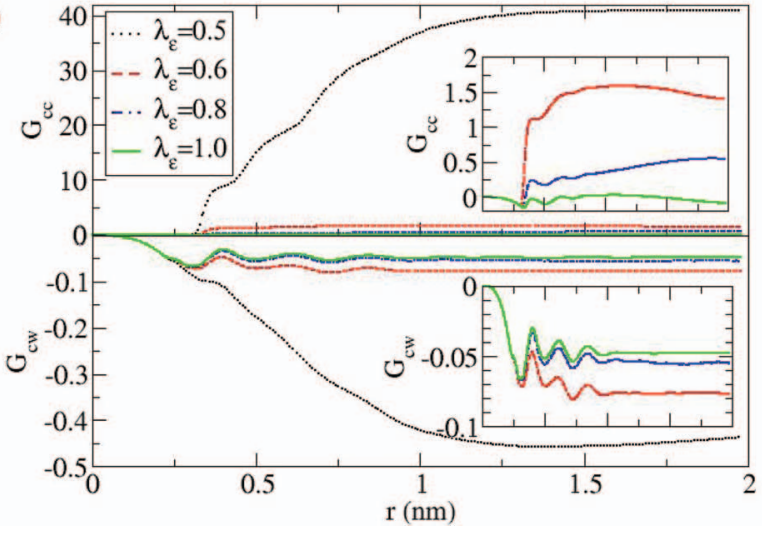

FIG. 1. (a) Radial distribution functions for force field Cs9I1 at $0.31 \mathrm{~m}$ and four different values of the LJ energy scaling factor $\lambda_{\varepsilon}$. Dotted(black), dashed(red), dot-dashed(blue), and solid(green) lines correspond to values of $\lambda_{\varepsilon} 0.5,0.6,0.8$, and 1.0, respectively. The different RDFs for all components of the solution, anions, cations, and water are shown. (b) The different Kirkwood-Buff integrals for Cs9I1 at $0.31 \mathrm{~m}$ and four different parameters of the scaling factor $\lambda_{\varepsilon}$ as a function of the upper integral boundary. Lines, colors and labeling are the same as in panel (a). (c) The composite Kirkwood-Buff integrals $G_{c c}$ and $G_{c w}$ as defined in Eq. (5) for Cs9I1 at $0.31 \mathrm{~m}$, which enter the calculation of the activity derivative in Eq. (4a). The curves are shown again for four different values of $\lambda_{\varepsilon}$, with line and color coding as in panel (a). The insets zoom into the values for $\lambda_{\varepsilon}=0.6,0.8,1.0$. All Kirkwood-Buff integrals are given in units $\mathrm{nm}^{3}$. salt-salt contribution, is positive, while the water-salt contribution, $G_{c w}$, is negative. For small $\lambda_{\varepsilon}$ prefactors, the ionic clustering leads to dramatic changes of the KBIs. For larger values of $\lambda_{\varepsilon}$ the variations of $G_{c c}$ and $G_{c w}$ are milder, as shown in the inset.

\section{B. Electrolyte activity derivatives}

We next proceed to the calculation of the activity derivative following the recipe of Eq. (4a). The experimental activities as a function of molality are obtained from Ref. 64. From these, the activity derivatives are extracted through Eq. (4b). Note, that the Kirkwood-Buff integrals depend on the RDFs and thus inherently include an error dependent on the statistical sampling in the MD simulations. We have estimated the error of the activity derivative $a_{c c}$ through block averaging of the MD data with a total time of $150 \mathrm{~ns}$ in blocks of $10 \mathrm{~ns}$ duration. The error follows from the variance of the block averages of the respective activity derivative $a_{c c}^{i}$ as error $^{2}=\sum_{i=1}^{m}\left(a_{c c}^{i}-\left\langle a_{c c}\right\rangle\right)^{2} /(m(m-1))$, where $\left\langle a_{c c}\right\rangle$ is the average value of the whole trajectory. More details can be found in Ref. 65. The error estimate for $\mathrm{KCl}$ and $\lambda_{\varepsilon}=1$ at $0.3 \mathrm{~m}$ is $a_{c c}=0.89 \pm 0.03$, as shown in Fig. 2(b). Only one error bar is shown for clarity, since it is similar for other data points as well. The results are summarized in Fig. 2, for $\mathrm{Na}^{+}, \mathrm{K}^{+}$, and $\mathrm{Cs}^{+}$salt solutions, respectively. In these figures, the lines correspond to experimental activity derivatives and data points denote MD simulation results. We first focus on the $\mathrm{Na}$ salts, $\mathrm{NaF}, \mathrm{NaCl}, \mathrm{NaBr}$, and $\mathrm{NaI}$. Fig. 2(a), shows that the MD data approach the experimental values above a threshold value of $\lambda_{\varepsilon}$. For $\mathrm{NaF}, \lambda_{\varepsilon}$ close to 1.5 matches the experimental value, for $\mathrm{NaCl}$ a value $\lambda_{\varepsilon}=1$ is suggested, and for $\mathrm{NaBr}, \lambda_{\varepsilon}=1$ gives the best comparison. For NaI, we use two different $\mathrm{I}^{-}$force fields (see Table I), a value of $\lambda_{\varepsilon}$ $=0.7$ matches the experimental data for $\mathrm{NaI} 1$ and $\lambda_{\varepsilon}=0.9$ for NaI4. The general trend that emerges is that for larger anions a smaller value of $\lambda_{\varepsilon}$ is suggested. We also show results for $\mathrm{NaCl}$ using a different KBT-derived force field. ${ }^{42}$ In contrast to our strategy, in that work the authors used a scaling factor 0.75 in the water-cation interaction parameter $\varepsilon$. The agreement with our result is good as expected, and serves as a check on our simulation methodology (we did not check the solvation free energy of that force field, where one would expect a more pronounced deviation). In the inset of Fig. 2(a) we show pictures of all ion pairs involving $\mathrm{Na}$, where the cation size has been increased by $0.04 \mathrm{~nm}$ with respect to the bare Pauling radii, as will be discussed further below. According to the heuristic law of matching water affinities, ${ }^{66}$ ions of similar radius interact more favorably with each other in water, and therefore are expected to have a smaller activity derivative $a_{c c}$, while ions with dissimilar radii interact less favorably and have a corresponding higher $a_{c c}$. In the inset of Fig. 2(a) the cation-anion RDFs for NaF5 and NaI4 are shown, which constitute an example of how an increasing radius mismatch between anions and cations is reflected in the distribution structure and specifically in the relative weight of the contact and solvent-separated pairing: while the RDF of $\mathrm{NaF}$ (which have well matching radii) is generally higher than the RDF of $\mathrm{NaI}$, the relative weight of contact and solvent-separated pairing is 
(a)

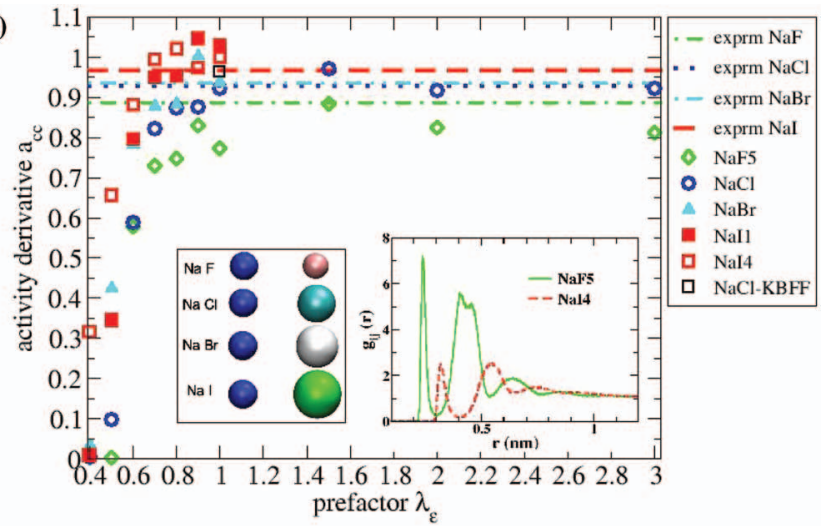

(b)

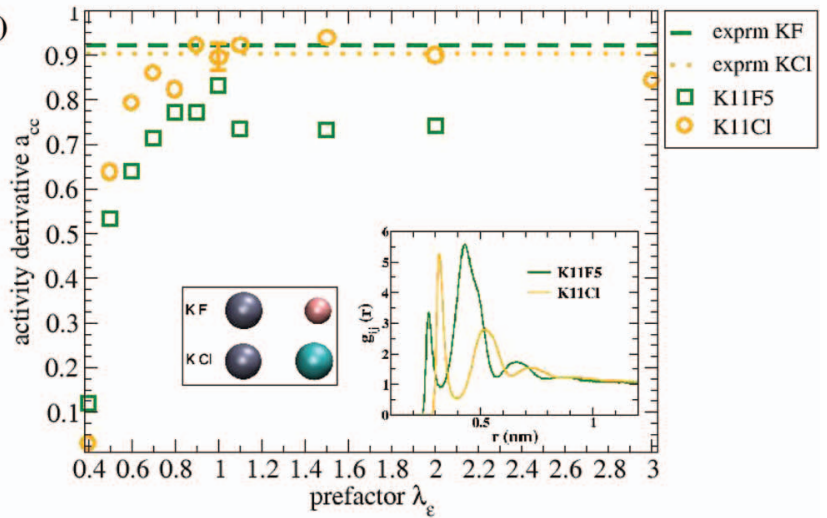

(c)

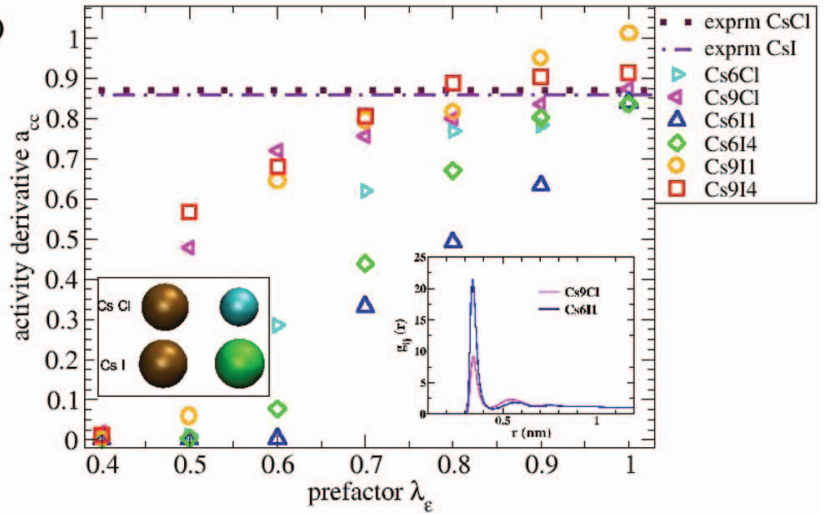

FIG. 2. Results for the activity derivative $\mathrm{a}_{c c}$ for different values of the scaling prefactor $\lambda_{\varepsilon}$ at $0.31 \mathrm{~m}$ for (a) the $\mathrm{Na}^{+}$-salts, (b) the $\mathrm{K}^{+}$-salts, and (c) the $\mathrm{Cs}^{+}$-salts, respectively. The lines are the respective experimental data as denoted by the labels, the data points denote simulation results. In all panels, the inset image shows the sizes of all ion-pairs with all cation radii increased by $0.04 \mathrm{~nm}$ (see text for discussion), while the inset graph shows two representative RDFs, for $\lambda_{\varepsilon}=1$ (see text). In panel (a), the label KBBF denotes an alternative Kirkwood-Buff-derived force field ${ }^{42}$ (see text). In panel (c), results are shown for all combinations of two force field sets for $\mathrm{Cs}^{+}$and $\mathrm{I}^{-}$. In panel (b) the error bar is shown only for one data point for clarity. The error bar is similar for all data points.

very similar. We will later observe that this is only one mechanism of how the ionic distribution function changes when the radius mismatch is increased.

Results for the $\mathrm{K}^{+}$salt solutions, $\mathrm{KCl}$ and $\mathrm{KF}$, using the $\mathrm{K} 11$ and F5 parameter sets, are shown in Fig. 2(b). For KCl, a good match between MD and experimental data is obtained for $\lambda_{\varepsilon}=1$. For KF, $\lambda_{\varepsilon}=1.0$ gives the closest match with the experimental data but shows a considerable residual deviation. Since the simulation curve for $a_{c c}$ as a function of $\lambda_{\varepsilon}$ shows a plateau, it is clear that $\varepsilon$ is not a good parameter to fix this problem and we shall return to KF in the next section. In the inset we show the RDFs for the two ion pairs involving potassium, demonstrating that the change in activity derivative here is brought about by a switch in the relative weight of the two peaks in the RDF and thus different from the $\mathrm{Na}$ case in Fig. 2(a). Whether the problems in reproducing the correct activity derivative of KF is related to the RDF structure and the dominance of the solvent-separated peak is not clear, though.

Data for Cs salt solutions are shown in Fig. 2(c). For $\mathrm{CsCl}$ the results for two different $\mathrm{Cs}^{+}$parameters are shown, while four different curves for CsI are depicted, including all different pairings of the $\mathrm{Cs}^{+}$and $\mathrm{I}^{-} \mathrm{LJ}$ parameters listed in Table I. For $\mathrm{Cs} 6 \mathrm{Cl}, \lambda_{\varepsilon}=1$ gives the best agreement, while for $\mathrm{Cs} 9 \mathrm{Cl}$ good agreement is seen for a value between $\lambda_{\varepsilon}=0.9$ and $\lambda_{\varepsilon}$ $=1$. For CsI, agreement with experiments is best for $\lambda_{\varepsilon}$ in the range between 0.8 and 1. Different combinations of $\mathrm{Cs}^{+}$and $\mathrm{I}^{-}$LJ parameters show variant behavior; both CsI solutions modeled with the Cs6 parameter set give an activity derivative that is almost zero up to $\lambda_{\varepsilon}=0.6$, beyond which they show an abrupt increase, with $\lambda_{\varepsilon}=1.0$ giving the best match for both Cs6I1 and Cs6I4. For the Cs9 parameter the curves increase more smoothly, and for both Cs9I4 and Cs9I1 $\lambda_{\varepsilon}=0.8$ leads to an $\mathrm{a}_{c c}$ value which is close to the experimental value. There is an intriguing reversal of the $\mathrm{a}_{c c}$ vs. $\lambda_{\varepsilon}$ curves for CsI in Fig. 2(c). The combination of the Cs6 parameter with I1 leads to a curve that is overall lower than that for Cs6I9. However, when combining Cs9 with $\mathrm{I} 1$, the respective curve is higher than the one for Cs9I4. In this sense, no clear decision can be made on which of the $\mathrm{Cs}^{+}$and $\mathrm{I}^{-} \mathrm{LJ}$ force fields are better optimized. All four LJ parameter sets can be favorably combined but lead to different $\lambda_{\varepsilon}$ scaling prefactors. The inset graph for the RDFs shows yet a different mechanism for a change in the activity derivative by solely decreasing the direction pair weight without modifying the solvent-separated ion peak.

As a general remark for all the salt solution data shown in Fig. 2, small $\lambda_{\varepsilon}$ values below 0.7 do not lead to thermodynamic properties that compare well to the respective experimental data. Most of the $a_{c c}$ data increase with $\lambda_{\varepsilon}$ and reach a plateau for large $\lambda_{\varepsilon}$. We also checked values of $\lambda_{\varepsilon}$ up to 3.0 for some test cases, like $\mathrm{NaCl}, \mathrm{NaF}, \mathrm{KF}, \mathrm{KCl}$, and verified that the activity derivatives seem to saturate or even decrease for very large $\lambda_{\varepsilon}$ values. Note that the radius increment of $0.04 \mathrm{~nm}$ for the cations used for the schematic pictures of ions in Fig. 2 is adjusted according to the activity coefficient derivatives and simply serves to graphically illustrate the law of matching water affinities, as will be discussed further below. As a result of this adjustment, ions of similar radius interact more attractive in water and thus have a lower activity derivative. Such an ion pairing has also been evident from molecular simulations and the respective potentials of mean force for the full set of alkali halide ion pairs. ${ }^{67}$

The force fields used in our current study and shown in Table I were taken from our previous study where they were found to optimally reproduce experimental osmotic coefficients. ${ }^{48}$ In that work, the osmotic coefficient was only determined approximately. In order to test whether the force 


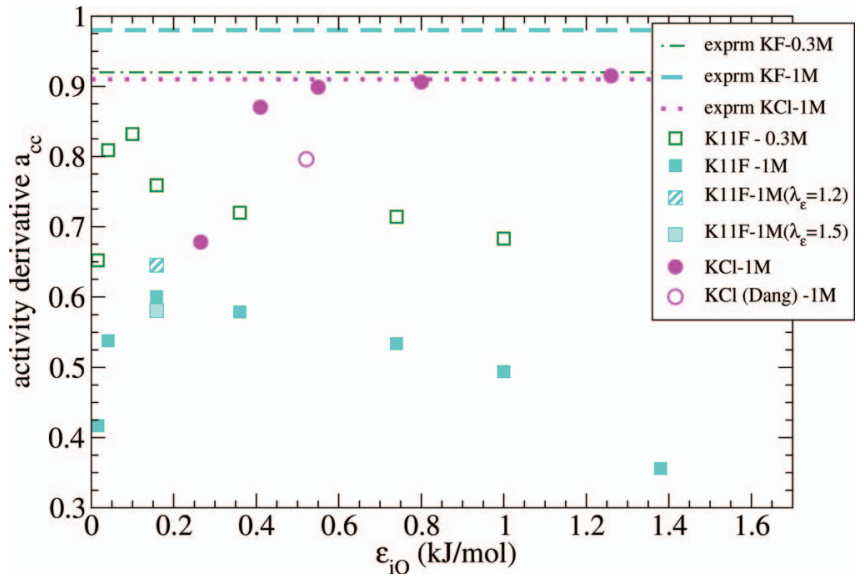

FIG. 3. Activity derivatives $\mathrm{a}_{c c}$ for the salts $\mathrm{KF}$ and $\mathrm{NaF}$ as a function of the $\mathrm{LJ}$ ion-water energy parameter $\varepsilon_{i O}$ with $\mathrm{LJ}$ radius $\sigma_{i O}$ constrained to the line on which the experimental ion solvation free energy is reproduced, similar to our previous study in Ref. 48. For K11F data the K11 force field is fixed and $\varepsilon_{F O}$ is varied, for $\mathrm{KCl}$ the $\mathrm{Cl}$ force field is fixed and $\varepsilon_{K O}$ is varied. The horizontal lines correspond to the experimental values for $\mathrm{KCl}$ and $\mathrm{KF}$. The results are shown for $1.03 \mathrm{~m}(1 \mathrm{M})$ as in Ref. 48. For KF, results are also shown for $0.31 \mathrm{~m}(0.3 \mathrm{M})$. All data are given for unmodified mixing rules, i.e., $\lambda_{\varepsilon}=1$, except for $\mathrm{K} 11 \mathrm{~F}$ at $1 \mathrm{M}$ with $\varepsilon_{F O}=0.1585 \mathrm{~kJ} / \mathrm{mol}$, where additional data for the values $\lambda_{\varepsilon}=1.2$ and $\lambda_{\varepsilon}=1.5$ are shown (cyan filled squares).

fields are optimal also when compared with experimental activity coefficients, in Fig. 3 we show the activity derivative $a_{c c}$ as a function of the ion LJ energy parameter $\varepsilon$. We picked $\mathrm{KCl}$, for which the $\mathrm{K}^{+} \mathrm{LJ}$ parameters $\varepsilon$ is varied, while the $\mathrm{Cl}$ parameters are fixed to the values given in Table I, and KF, for which $\mathrm{K}^{+}$is fixed to the $\mathrm{K} 11$ force field values in Table I and the $\mathrm{LJ} \varepsilon$ parameter for $\mathrm{F}^{-}$is varied. The variation of both $\mathrm{LJ}$ parameters for $\mathrm{K}^{+}$and $\mathrm{F}^{-}$follow the free energy of solvation curves for these ions. ${ }^{29}$ For KF we present results for $0.31 \mathrm{~m}$ and $1.03 \mathrm{~m}(1 \mathrm{M})$, all other data in Fig. 3 are for $1.03 \mathrm{~m}(1 \mathrm{M})$; note that all other results in this work are for $0.31 \mathrm{~m}(0.3 \mathrm{M})$. Most data in the figure are for $\lambda_{\varepsilon}=1$, for $\mathrm{KF}$ at $1 \mathrm{M}$ data for two additional $\lambda_{\varepsilon}$ values are shown. As the data show, the force fields found previously to be optimal when compared with experimental osmotic coefficient data, namely K11 with $\varepsilon_{K O}=1.26 \mathrm{~kJ} / \mathrm{mol}$ and F5 with $\varepsilon_{F O}=0.1 \mathrm{~kJ} / \mathrm{mol}$, are also found to be optimal in the present comparison based on activity coefficients. In agreement with our previous findings, for $\mathrm{KF}$, no $\mathrm{F}$ parameter gives perfect match with the experimental data at $0.31 \mathrm{~m}$ and $1.03 \mathrm{~m}$. The data for $\mathrm{K} 11 \mathrm{~F}$ at $1 \mathrm{M}$ for $\varepsilon_{F O}$ $=0.1585 \mathrm{~kJ} / \mathrm{mol}$ show similar trends as the data presented in Fig. 2(b) for $0.31 \mathrm{~m}$ : an increase in $\lambda_{\varepsilon}$ cannot yield perfect agreement with experimental data since the simulation results for $a_{c c}$ show a maximum with increasing $\lambda_{\varepsilon}$.

\section{A problematic case: Fluoride}

Evidently, the most problematic case among the salt solutions studied here is $\mathrm{KF}$. For $\mathrm{NaF}$, as demonstrated in Fig. 2(a), the scaling parameter $\lambda_{\varepsilon}$ should be around 1.5 in order to obtain reasonable match with experimental data. This is in contrast to the case of iodide and means that a larger LJ repulsion at low distances is needed. However, when fluoride pairs with potassium, as seen in Fig. 2(b), even values

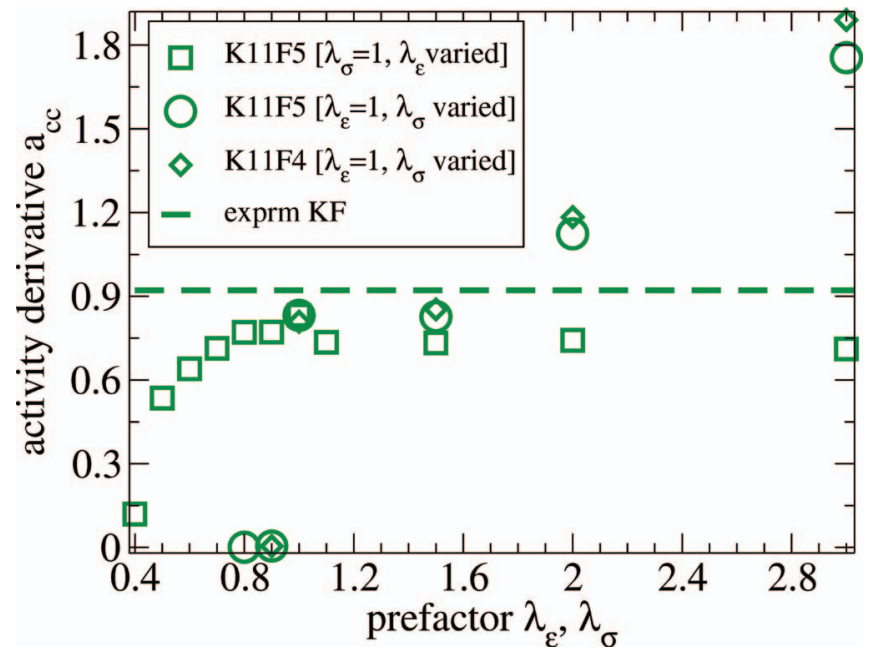

FIG. 4. Activity derivatives $\mathrm{a}_{c c}$ for the KF solutions as a function of the scaling factors $\lambda_{\varepsilon}$ and $\lambda_{\sigma}$. Note that only one of the factors is varied, the other is fixed at unity. The line shows the respective experimental value. The concentration is $0.31 \mathrm{~m}(0.3 \mathrm{M})$.

$\lambda_{\varepsilon} \gg 1$ do not give results closer to the experimental data since the activity derivative shows a maximum as a function of $\lambda_{\varepsilon}$. We will explain this finding further below. For the moment, we use the unmodified mixing rule $\left(\lambda_{\varepsilon}=1\right)$ for the $\varepsilon_{+-}$ LJ cation-anion interaction parameter but modify the mixing rule for the $\mathrm{LJ}$ diameter $\sigma_{+-}$according to $\sigma_{+-}=\lambda_{\sigma}\left(\sigma_{++}\right.$ $\left.+\sigma_{--}\right) / 2$. With this approach, we focus on KF, shown in Fig. 4. We use for this again the F5 force field and also an additional force field for $\mathrm{F}$, with a small $\mathrm{LJ}$ interaction energy, for comparison. We denote this additional parameter set, with values $\varepsilon_{i O}=0.041 \mathrm{~kJ} / \mathrm{mol}$ and $\sigma_{i O}=0.38 \mathrm{~nm}$, as F4 (according to our previous study ${ }^{48}$ ). Values $\lambda_{\sigma}<1$ lead to ion clustering and activity derivatives close to zero. However, for increasing $\lambda_{\sigma}$, the simulated activity derivatives keep increasing, and cross the experimental line around $\lambda_{\sigma}=1.5 \mathrm{~K} 11 \mathrm{~F} 4$ and K11F5 force fields show similar behavior. As a comparison, we show again the data for K11F5 for fixed $\lambda_{\sigma}=1$ as a function of $\lambda_{\varepsilon}$. It is clearly seen that the scaling factor $\lambda_{\sigma}$ for the cation-anion LJ diameter $\sigma_{+-}$term is a much more efficient optimization parameter than the scaling factor $\lambda_{\varepsilon}$ for the $\varepsilon_{+-} \mathrm{LJ}$ energy. The reason for this is most probably that fluoride is the smallest anion and solvates water very strongly, which becomes problematic when ion-pairing with the relatively large cation $\mathrm{K}$ is considered. In this case, the use of the $\lambda_{\sigma}$ scaling factor is necessary. This is in contrast to the other salts studied here, for which a scaling in the cation-anion interaction term $\varepsilon_{+-}$was sufficient.

The optimal $\lambda_{\varepsilon}$ scaling prefactors in the cation-anion interaction as obtained by the comparison of the MD data to the experimental counterparts (see Fig. 2) are summarized in Table II. These values are obtained from the activity derivative data shown in Figs. 2 and 4 and correspond to the interpolated crossing points of the MD data with the respective experimental values, denoted by horizontal lines in the figure. The symbols in the parentheses denote the specific force fields used for cases where more than one force field was studied, e.g., $\mathrm{NaI}$, for which both I1 and I4 force fields were considered. As is 
TABLE II. Optimal $\lambda_{\varepsilon}, \lambda_{\sigma}$ scaling prefactors for the cation-anion combinations studied in this work.

\begin{tabular}{lccc}
\hline \hline & \multicolumn{3}{c}{$\lambda_{\varepsilon}$} \\
\cline { 2 - 4 } & $\mathrm{Na}^{+}$ & $\mathrm{K}^{+}$ & $\mathrm{Cs}^{+}$ \\
\hline $\mathrm{F}^{-}$ & 1.5 & $\mathrm{X}$ & $\ldots$ \\
$\mathrm{Cl}^{-}$ & 1.0 & 1.0 & $1.0(\mathrm{Cs} 6), 1.0(\mathrm{Cs} 9)$ \\
$\mathrm{Br}^{-}$ & 1.0 & $\ldots$ & $\ldots$ \\
$\mathrm{I}^{-}$ & $0.8(\mathrm{I} 4), 0.9(\mathrm{I} 1)$ & $\ldots$ & $1.0(\mathrm{Cs} 611, \mathrm{Cs} 6 \mathrm{I} 4)$, \\
& & & $0.85(\mathrm{Cs} 9 \mathrm{I} 1), 0.80(\mathrm{Cs} 9 \mathrm{I} 4)$ \\
& & $\mathrm{K}_{\sigma}$ & $\mathrm{Ks}^{+}$ \\
$\mathrm{F}^{-}$ & $\ldots$ & $1.65(\mathrm{~F} 5), 1.65(\mathrm{~F} 4)$ & $\ldots$ \\
\hline \hline
\end{tabular}

evident from Table II, for the $\mathrm{Cl}^{-}$and $\mathrm{Br}^{-}$salts studied in this work, $\lambda_{\varepsilon} \simeq 1$ is a value that correctly reflects the cationanion interaction. This value corresponds to the unmodified Lorentz-Berthelot mixing rule and verifies our previously optimized force fields for these monovalent ions. ${ }^{48}$ The situation is different for the $\mathrm{F}^{-}$and $\mathrm{I}^{-}$salts, which often have been proven difficult to deal with. ${ }^{29,48,57}$ In the case of $\mathrm{F}^{-}$, values of $\lambda_{\varepsilon}$ greater than 1 lead to a better match of the experimental data, but for KF changing $\lambda_{\varepsilon}$ alone is not sufficient and in addition we had to scale the cation-anion $\sigma_{+-}$parameter.

An explanation of this finding is given in Fig. 5, where we show radial distribution functions for K11F5 for different $\lambda_{\varepsilon}$ and $\lambda_{\sigma}$ factors. For the unmodified case $\lambda_{\varepsilon}=1$ and $\lambda_{\sigma}=1$, shown at the top, the direct-ion pairing state and the solventseparated pairing state are both populated. The activity coefficient derivate for the force field is too low when compared to experiments (see Figs. 2(b) and 4), meaning that the RDF should be smaller. By increasing $\lambda_{\varepsilon}$ alone, as shown to the left, the direct-ion pairing state is depopulated due to the increasingly repulsive component of the $\mathrm{LJ}$ interaction, but the $\mathrm{LJ}$ depth at a distance corresponding to the solvent-separated pair increases and thus enhances the RDF in this distance range (the LJ potentials and the total cation-anion interaction energy

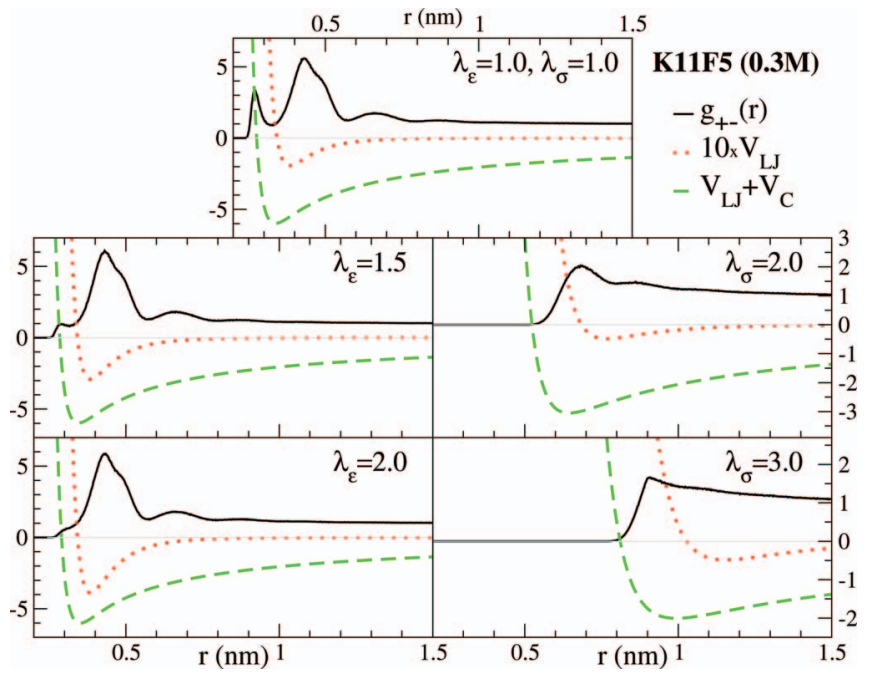

FIG. 5. Cation-anion radial distribution functions $g_{+-}$, Lennard-Jones potentials $V_{L J}$, and total (Lennard-Jones plus Coulomb) potentials $V_{L J}+V_{C}$ for the salt K11F5 at $0.31 \mathrm{~m}(0.3 \mathrm{M})$. Note that $V_{L J}$ (red dotted lines) has been magnified 10 times for clarity. The uppermost panel shows results for unmodified mixing rules with scaling factors $\lambda_{\varepsilon}, \lambda_{\sigma}=1.0$. The left and right panels correspond to the modifications $\lambda_{\varepsilon}>1$, and $\lambda_{\sigma}>1$, respectively, where the other scaling factor is unity.

including the Coulombic term are also shown in the figures). The two effects of the repulsive and the attractive LJ components more or less cancel, and therefore there is no clear net effect on the $\mathrm{KB}$ integrals and thus on the activity derivative. The situation is qualitatively different for increasing values of $\lambda_{\sigma}$, shown in the right panels of Fig. 5. Here, an increasing LJ diameter shifts the RDF to larger radii, for which the Coulombic attraction between anion and cation is smaller, and thus leads to an overall decrease of the RDF and thereby to an increase of the activity coefficient $a_{c c}$. In essence, changing the mixing rule for the LJ diameter constitutes an efficient and robust route to successful ion force field optimization.

The ionic force fields in Table I were obtained with unmodified mixing rules through a comparison of MD-derived

TABLE III. Excess coordination numbers for the optimized scaling prefactors given in Table II from our simulation results, denoted as $\mathrm{N}_{c c}^{M D}=\rho_{c} \mathrm{G}_{c c}$ and $\mathrm{N}_{c w}^{M D}=\rho_{w} \mathrm{G}_{c w}$, and experimental values as taken from the analysis in Ref. 23, denoted by $\mathrm{N}_{c c}^{\text {exp }}$ and $\mathrm{N}_{c w}^{\text {exp }}$. Most data are shown for $0.31 \mathrm{~m}$, numbers in parentheses correspond to solutions of $1 \mathrm{~m}$ concentration. The activity derivatives $a_{c c}^{M D}$ are computed through Eq. (4a) using the $\mathrm{N}_{c c}^{M D}$, $\mathrm{N}_{c w}^{M D}$ MD data, and can be compared to the experimental data given in Refs. 23 and 64, denoted as $\mathrm{a}_{c c}^{\text {exp, } 64}$ and $\mathrm{a}_{c c}^{\text {exp, } 23}$, respectively.

\begin{tabular}{|c|c|c|c|c|c|c|c|}
\hline Salt & $\mathrm{N}_{c c}^{M D}$ & $\mathrm{~N}_{c w}^{M D}$ & $\mathrm{a}_{c c}^{M D}$ & $\mathrm{~N}_{c c}^{e x p}($ Ref. 23) & $\mathbf{N}_{c w}^{\exp }($ Ref. 23) & $\mathrm{a}_{c c}^{\exp }($ Ref. 64) & $\mathrm{a}_{c c}^{\exp }($ Ref. 23) \\
\hline NaF5 & 0.14 & 0.56 & 0.88 & 0.15 & 0.08 & 0.89 & 0.87 \\
\hline $\mathrm{NaCl}$ & 0.08 & -0.26 & 0.92 & 0.09 & -0.47 & 0.93 & 0.92 \\
\hline $\mathrm{NaBr}$ & $0.06(-0.09)$ & $-0.96(-0.72)$ & $0.93(0.91)$ & $0.05(-0.08)$ & $-0.66(-0.62)$ & $0.94(1.04)$ & $0.94(1.05)$ \\
\hline NaI1 & 0.05 & -0.87 & 0.94 & 0.02 & -0.96 & 0.97 & 0.98 \\
\hline $\mathrm{NaI} 4$ & $-0.03(-0.27)$ & $-1.03(-1.01)$ & $1.02(1.35)$ & $0.02(-0.13)$ & $-0.96(-0.83)$ & $0.97(1.07)$ & $0.98(1.11)$ \\
\hline KF5 & 0.11 & 0.42 & 0.90 & $\ldots$ & $\ldots$ & 0.92 & $\ldots$ \\
\hline KF4 & 0.08 & 0.20 & 0.93 & $\ldots$ & $\ldots$ & 0.92 & $\ldots$ \\
\hline $\mathrm{KCl}$ & 0.11 & -0.74 & 0.89 & 0.11 & -0.80 & 0.90 & 0.91 \\
\hline $\mathrm{Cs} 6 \mathrm{Cl}$ & 0.18 & -1.01 & 0.84 & 0.14 & -1.22 & 0.87 & 0.87 \\
\hline $\mathrm{Cs} 9 \mathrm{Cl}$ & 0.13 & -1.01 & 0.88 & 0.14 & -1.22 & 0.87 & 0.87 \\
\hline Cs6I1 & 0.17 & -1.80 & 0.84 & $\ldots$ & $\ldots$ & 0.86 & \\
\hline Cs6I4 & 0.17 & -2.00 & 0.84 & $\ldots$ & $\ldots$ & 0.86 & $\ldots$ \\
\hline Cs9I1 & 0.06 & -1.67 & 0.93 & $\ldots$ & $\ldots$ & 0.86 & $\ldots$ \\
\hline Cs9I4 & 0.11 & -1.78 & 0.88 & $\ldots$ & $\ldots$ & 0.86 & $\ldots$ \\
\hline
\end{tabular}



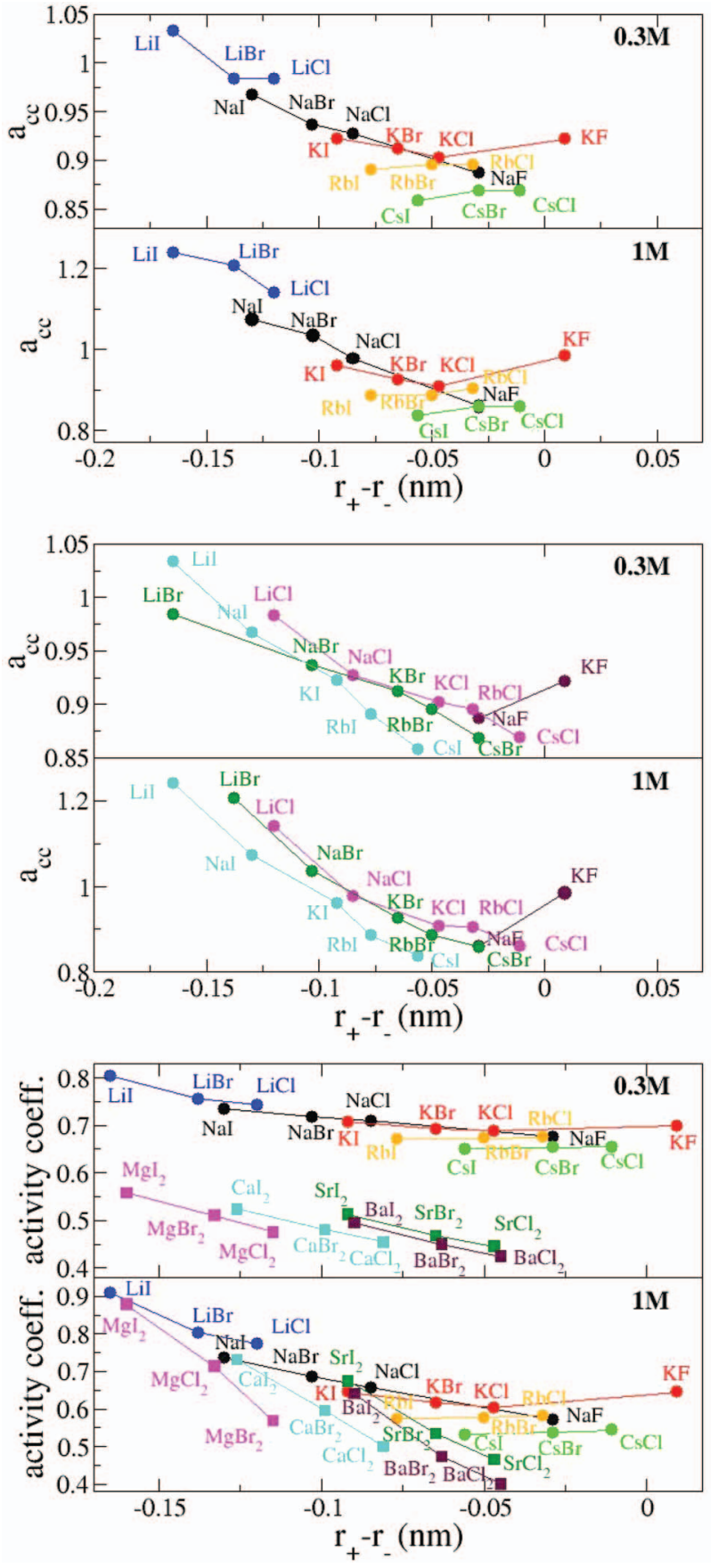

(c)

FIG. 6. (a) Experimental activity derivatives $\mathrm{a}_{c c}$ as a function of the difference $r_{+}-r_{-}$, where $r_{+}$and $r_{-}$denote the Pauling radii of the cation and anion, respectively. All salt solutions considered in this work are shown at concentrations of $0.31 \mathrm{~m}(0.3 \mathrm{M})$ (upper panel) and $1.03 \mathrm{~m}(1 \mathrm{M})$ (lower panel), respectively. The color-coding is for fixed cations. (b) Same as in (a), except for the color-coding which corresponds to fixed anions. (c) Experimental activity coefficients as a function of $r_{+}-r_{-}$for different monovalent and divalent salt solutions at $0.31 \mathrm{~m}(0.3 \mathrm{M})$ (upper panel) and $1 \mathrm{~m}$ (lower panel) concentration.

osmotic coefficients with experimental values ${ }^{48}$ and served as a starting point for our present investigation, where we have used the activity derivative as a probe to optimize the cation-anion combination rules. In order to check whether additional observables that were not considered in the optimization match experimental results, we calculate the excess coordination numbers $N_{c c}, N_{c w}$ obtained directly from the Kirkwood-Buff integrals. For the optimized values of $\lambda_{\varepsilon}$ given in Table II, we show the resulting values for $\mathrm{N}_{c c}, \mathrm{~N}_{c w}$ at $0.31 \mathrm{~m}$ in Table III, some results for $1.03 \mathrm{~m}$ concentration are shown in parentheses. Experimental data analyzed in Ref. 23 are shown in the two rightmost columns.

Inspection of the data shows that the overall agreement is quite good and for some cases $(\mathrm{NaBr}, \mathrm{NaI})$ even better than the Kirwood-Buff derived force field (KBFF) parameters of Ref. 23. However, there are also some cases, in particular $\mathrm{N}_{c w}$ for $\mathrm{NaCl}, \mathrm{NaF}$, and $\mathrm{CsCl}$, for which the error is larger. Note, that we have optimized the activity derivative $\mathrm{a}_{c c}$, which probes the difference $\rho_{c}\left(G_{c c}\right.$ $\left.-G_{c w}\right)=N_{c c}-\left(\rho_{c} / \rho_{w}\right) N_{c w}$ with respect to experimental data. In essence, if $a_{c c}$ and $N_{c c}$ both match experiments, then $N_{c w}$ also must agree. The problem of course lies in the prefactor $\left(\rho_{c} / \rho_{w}\right)$ which is very small and makes the comparison of $N_{c w}$ unreliable. In order to understand the discrepancies for $N_{c w}$ shown in the table better, we have computed $a_{c c}$ using Eq. (4a) and the MD values $\mathrm{N}_{c c}^{M D}$, and $\mathrm{N}_{c w}^{M D}$ shown in Table III. We then compare the computed $\mathrm{a}_{c c}\left(\mathrm{a}_{c c}^{M D}\right.$ in Table III) to the respective experimental data given in Refs. 23 and 64 $\left(\mathrm{a}_{c c}^{e x p, 23}\right.$ and $\mathrm{a}_{c c}^{e x p, 64}$ in Table III). One clearly sees that there are only statistically insignificant deviations in $a_{c c}$ between the two experimental data sets and the MD simulations on the other hand. Because of the above-mentioned statistical error in the values of $N_{c w}$, one should therefore at the present stage mostly consider the results for $N_{c c}$, for which the agreement between experiments and simulations is satisfactory.

\section{SUMMARY AND CONCLUSIONS}

We have used molecular dynamics simulations to optimize ionic force-field parameters in conjunction with the SPC/E water model. Seven different ions and a total of eight ion-pairs were investigated. Our study was based on previously determined ionic LJ force fields for which the experimental free energy of solvation is reproduced. We have introduced scaling factors for the cation-anion LJ interaction that quantify deviations from the standard mixing rules, determined the RDFs for each ion pair as a function of the cationanion scaling factor and calculated the KB integrals. Using the well-known link between KBIs and the activity derivatives, we could directly compare simulations to experimental data. We have verified that with modified cation-anion mixing rules, ionic force fields that reproduce solution activities with invariant ion-water interaction parameters can be constructed.

The resultant behavior can be most conveniently classified with respect to the anions. For the bromide and chloride solutions modeled here, $\lambda_{\varepsilon}=1$ is the best choice for maximizing the agreement of the MD activity derivatives with the experimental ones. This leads back to the unmodified mixing rules and our previously optimized ionic force fields. ${ }^{48}$ On the other hand, for the iodide and fluoride solutions, corresponding to the largest and smallest anion considered in this work, a rescaling of the mixing rules was necessary. For iodide, the unmodified mixing rule produced $\mathrm{KB}$ integrals that show not enough attraction between iodide and the respective cation, leading to a too large activity coefficient derivative. This finding might reflect attractive charge-induced dipole interaction 
between the ions, in line with the large excess polarizability of the large halides. ${ }^{68-70}$ In any case, this problem can be easily remedied by reducing the scaling factor of the cation-anion $\mathrm{LJ}$ energy, $\lambda_{\varepsilon}$. For the smallest anion, $F^{-}$, the situation is different and the simulations show too large attraction between fluoride and cations, possibly reflecting the fact that fluoride has a small excess polarizability. ${ }^{68-70}$ For $\mathrm{NaF}$ the experimental activity coefficient derivative could be matched by choosing a scaling factor $\lambda_{\varepsilon}$ of the order of 1.5. For KF, on the other hand, this procedure did not work since the KB integral saturates with increasing $\lambda_{\varepsilon}$, which is explained by enhanced $\mathrm{LJ}$ attraction of the solvent-separated ion pair. In order to resolve this, we modified the cation-anion LJ diameter $\sigma_{+-}$by a scaling prefactor $\lambda_{\sigma}$. The optimal value of $\lambda_{\sigma}$ for KF was found to be of the order of 1.5. Although not considered by us in this work, similar behavior is also expected for the smallest cation, $\mathrm{Li}^{+},{ }^{23}$ which could be easily counteracted by increasing the effective $\mathrm{LJ}$ diameter of the ion pair through the $\lambda_{\sigma}$ prefactor in order to be able to correctly reproduce the thermodynamic properties of the solution.

Our ionic force field optimization approach based on modifying the cation-anion mixing rules is robust and can be efficiently transferred to other salt solutions as well. Our aim in this study was not to span an exhaustive set of anions and cations or salt concentrations, but rather to propose a general pathway to optimize ionic force fields simultaneously based on infinite-dilution singe-ion properties (here taken as solvation free energies) and finite-concentration ion-pair properties (here taken to be activity derivatives as determined by KB integrals). Transferability to different ion pairs is not really an issue in our current treatment, since the LJ parameters for each ion pair should be adjusted separately if the corresponding KB integral indicates departures from the experimental values. Such departures are particularly expected for ion pairs with large size mismatches. Another issue is the applicability of the optimization proposed here in reproducing other properties, for example properties in the crystalline state such as lattice energies and lattice constants or dynamic properties such as ion-pair association times. In this paper, we have confined ourselves to thermodynamic properties and based our optimization on the solution activity but also showed that excess coordination numbers as well as osmotic coefficients ${ }^{48}$ are also reproduced quite well. This means that the force fields derived here should work whenever thermodynamic pair properties in solution are considered. There is no reason why the crystalline state should be equally well described, so if in an application the correct equilibrium between an ionic solution and a salt crystal is important, a different optimization strategy should be employed.

It is, finally, known that classical pairwise ionic force fields have fundamental drawbacks compared to accurate quantum mechanical ones. Often one needs to use more complicated interaction potentials or polarizable interaction potentials. However, we in this paper have shown that some of the effects of ionic polarizability, presumably at the heart of the deviations from the standard mixing rules seen for iodide and fluoride, can be included by modification of the ion-pair mixing rules. We reiterate that there is no reason why the heuristic law of matching water affinities, which is related to the simple fact that small ions have a tightly bound water hydration shell, while hydration water around large ions can be easily displaced, should not be reproduced - at least qualitatively - by the standard mixing rules in simulations with explicit water. The deviations from the standard mixing rules, which is the main focus of the present communication, are fine details and do not contradict the basic law of matching water affinities. The best graphical representation is obtained when the cation Pauling radii are increased by $0.04 \mathrm{~nm}$. This becomes clear when looking at the activity derivative $a_{c c}$ as a function of the radius difference $r_{+}-r_{-}$, where $r_{+}$and $r_{-}$denote the cation and anion Pauling radii, respectively. The data for $a_{c c}$ roughly show a minimum around $r_{+}-r_{-}=-0.04 \mathrm{~nm}$, as shown in Fig. 6(a). Although the KB integrals, as an integral over the RDF, form a very robust and discriminating measure of cation-anion correlations, they are insensitive to fine details of the detailed ion-distribution. This was exemplified by the various RDFs shown as insets in the three panels in Fig. 2, showing that changes in the KB integral can be brought about by different mechanisms, e.g., a global decrease of the RDF (comparing NaF and NaI in Fig. 2(a)), an exchange of the predominance of the direct and solventseparated ion pairing (comparing $\mathrm{KF}$ and $\mathrm{KCl}$ in Fig. 2(b)), or a change in the direct ion-pairing peak without changing the population of the solvent-separated peak (comparing $\mathrm{CsCl}$ and CsI in Fig. 2(c)); other more complicated mechanisms are also conceivable. Note that there seems to be a slight asymmetry between cations and anions, because when plotting $a_{c c}$ for different cations and fixed anions as in Fig. 6(b), where we only changed the color coding when compared to Fig. 6(a), a minimum in $a_{c c}$ is less prominent and in fact only visible for fluoride, the smallest anion. Finally, in Fig. 6(c) we show the activity coefficient itself, which for fixed monovalent cations shows also a slight minimum as a function of $r_{+}-r_{-}$. In this graph we also include data for divalent cations, which do not show a minimum in the available radius difference range and therefore seem to require a modification from the law of matching water affinities (when formulated in the context of activities).

\section{ACKNOWLEDGMENTS}

The authors wish to thank I. Kalcher, S. Mamatkulov, E. Schneck, and D. Horinek for useful discussions and S. Weeresanghe for an informative correspondence. Financial support is acknowledged from the German-Israeli Foundation for Scientific Research and Development (GIF), the 'Gender Issue Incentive Funds' of the Cluster of Excellence in Munich, Germany, and the 'BioFuS' project of the IntraEuropean Marie-Curie Programm (call FP7-PEOPLE-2009IEF).

${ }^{1}$ W. L. Jorgensen, J. Chandrasekhar, J. D. Madura, R. W. Impey, and M. L. Klein, J. Chem. Phys. 79, 926 (1983).

${ }^{2}$ F. Sedlmeier, D. Horinek, and R. R. Netz, J. Am. Chem. Soc. 133, 1391 (2011).

${ }^{3}$ K. M. Dyer, J. S. Perkyns, G. Stell, and B. M. Pettitt, Mol. Phys. 107, 423 (2009).

${ }^{4}$ W. L. Jorgensen, J. Am. Chem. Soc. 103, 335 (1981). 
${ }^{5}$ H. J. C. Berendsen, J. P. M. Postma, W. F. van Gunsteren, and J. Hermans, in Intermolecular Forces, edited by B. Pullman (Reidel, Dordrecht, 1981).

${ }^{6}$ H. J. C. Berendsen, J. R. Grigera, and T. P. Straatsma, J. Phys. Chem. 91, 6269 (1987).

${ }^{7}$ W. L. Jorgensen, J. Chem. Phys. 77, 4156 (1982).

${ }^{8}$ H. W. Horn, W. C. Swope, J. W. Pitera, J. D. Madura, T. J. Dick, G. L. Hura, and T. Head-Gordon, J. Chem. Phys. 120, 9665 (2004).

${ }^{9}$ J. L. F. Abascal, E. Sanz, R. Garca Fernández, and C. Vega, J. Chem. Phys. 122, 234511 (2005).

${ }^{10}$ J. L. F. Abascal and C. Vega, J. Chem. Phys. 123, 234505 (2005).

${ }^{11}$ F. H. Stillinger and A. Rahman, J. Chem. Phys. 60, 1545 (1974).

${ }^{12}$ M. W. Mahoney and W. L. Jorgensen, J. Chem. Phys. 112, 8910 (2000).

${ }^{13}$ S. W. Rick, J. Chem. Phys. 120, 6085 (2004).

${ }^{14}$ H. Nada and J. P. J. M. van der Eerden, J. Chem. Phys. 118, 7401 (2003).

${ }^{15}$ A. Kumar, Chem. Rev. 101, 1 (2001).

${ }^{16}$ W. Kunz, Pure Appl. Chem. 78, 1611 (2006).

${ }^{17}$ B. Alberts, A. Johnson, J. Lewis, M. Raff, K. Roberts, and P. Walter, Molecular Biology of the Cell (Garland Science, New York, 2002).

${ }^{18}$ A. Savelyev and G. A. Papoian, J. Phys. Chem. B 112, 9135 (2008).

${ }^{19}$ T. E. Cheatham III and M. A. Young, Biopolymers (Nucleic Acid Sci.) 56, 232 (2001).

${ }^{20}$ T. M. Chang and L. X. Dang, Chem. Rev. 106, 1305 (2006).

${ }^{21}$ P. Jungwirth and D. J. Tobias, Chem. Rev. 106, 1259 (2006).

${ }^{22}$ D. Frenkel and B. Smit, Understanding Molecular Simulations (Academic, New York, 1996).

${ }^{23}$ M. B. Gee et al., J. Chem. Theory Comput. 7, 1369 (2011).

${ }^{24}$ C. Krekeler and L. Delle Sitte, J. Phys.: Condens. Matter 19, 192101 (2007)

${ }^{25}$ J. N. Canongia Lopes, J. Deschamps, and A. A. H. Pádua, J. Phys. Chem. B 108, 2038 (2004).

${ }^{26}$ M. A. Young, G. Ravishanker, and D. L. Beveridge, Biophys. J. 73, 2313 (1997).

${ }^{27}$ D. E. Smith and L. X. Dang, J. Chem. Phys. 100, 3757 (1994).

${ }^{28}$ I. S. Joung and T. E. Cheatham, III, J. Phys. Chem. B 112, 9020 (2008).

${ }^{29}$ D. Horinek, S. Mamatkulov, and R. R. Netz, J. Chem. Phys. 130, 124507 (2009).

${ }^{30}$ A. A. Chen and R. V. Pappu, J. Phys. Chem. B 111, 11884 (2007).

${ }^{31}$ B. R. Brooks, R. E. Bruccoleri, B. D. Olafson, D. J. States, S. Swaminathan, and M. Karplus, J. Comput. Chem. 4, 187 (1983).

${ }^{32}$ S. Berneéche and B. Roux, Nature (London) 414, 73 (2001).

${ }^{33}$ S. Yu. Noskov, S. Bernéche, and B. Roux, Nature (London) 431, 830 (2004).

${ }^{34}$ Y. Luo and B. Roux, J. Phys. Chem. Lett. 1, 183 (2010).

${ }^{35}$ S. Weerasinghe and P. E. Smith, J. Chem. Phys. 119, 11342 (2003).

${ }^{36}$ B. Hess, C. Holm, and N. van der Vegt, Phys. Rev. Lett. 96, 127801 (2006).

${ }^{37}$ A. P. Lyubartsev and A. Laaksonen, Phys. Rev. E 55, 5689 (1997).
${ }^{38}$ J. G. Kirkwood and F. P. Buff, J. Chem. Phys. 19, 774 (1951).

${ }^{39}$ P. G. Kusalik and G. N. Patey, J. Chem. Phys. 86, 5110 (1987).

${ }^{40}$ R. Chitra and P. E. Smith, J. Chem. Phys. 114, 426 (2001).

${ }^{41}$ R. Chitra and P. E. Smith, J. Chem. Phys. 106, 1491 (2002).

${ }^{42}$ S. Weerasinghe and P. E. Smith, J. Chem. Phys. 118, 10663 (2003)

${ }^{43}$ D. Horinek and R. R. Netz, J. Phys. Chem. A 115, 6125 (2011).

${ }^{44}$ B. Hess and N. F. A. van der Vegt, Proc. Natl. Acad. Sci. U.S.A. 106, 13296 (2009).

${ }^{45}$ B. Klasczyk and V. Knecht, J. Chem. Phys. 132, 024109 (2010).

${ }^{46}$ P. L. Cummins and J. E. Gready, J. Comput. Chem. 15, 704 (1994).

${ }^{47}$ J. T. Wescott, L. R. Fisher, and S. Hanna, J. Chem. Phys. 116, 2361 (2002).

${ }^{48}$ M. Fyta, I. Kalcher, L. Vrbka, J. Dzubiella, and R. R. Netz, J. Chem. Phys 132, 024911 (2010).

${ }^{49}$ T.-M. Chang and L. X. Dang, J. Phys. Chem. B 103, 4714 (1999).

${ }^{50}$ S. Ramajani, T. Ghosh, and S. Garde, J. Chem. Phys. 120, 4457 (2004).

${ }^{51}$ L. X. Dang, J. Am. Chem. Soc. 117, 6954 (1995).

${ }^{52}$ L. X. Dang, J. Chem. Phys. 96, 6970 (1992).

${ }^{53}$ K. P. Jensen and W. L. Jorgensen, J. Chem. Theory Comput. 2, 1499 (2006).

${ }^{54}$ H. J. C. Berendsen, D. van der Spoel, and R. van der Drunen, Comput. Phys. Commun. 91, 43 (1995)

${ }^{55}$ E. Lindahl, B. Hess, and D. van der Spoel, Journal of Molecular Modeling 7, 306 (2001).

${ }^{56}$ H. J. C. Berendsen, J. P. M. Postma, W. F. van Gunsteren, A. DiNola, and J. R. Haak, J. Chem. Phys. 81, 3684 (1984).

${ }^{57}$ I. Kalcher and J. Dzubiella, J. Chem. Phys. 130, 134507 (2009).

${ }^{58}$ U. Essmann, L. Perera, M. L. Berkowitz, T. Darden, H. Lee, and L. G. Pedersen, J. Chem. Phys. 103, 8577 (1995).

${ }^{59}$ D. Frenkel and B. Smit, Understanding Molecular Simulation: From Algorithms to Applications (Academic, 1996).

${ }^{60}$ J. Dzubiella, M. Fyta, D. Horinek, I. Kalcher, R. R. Netz, and N. Schwierz, "Ion-specificity: from solvation thermodynamics to molecular simulations and back," in Specific Ion Effects, edited by W. Kunz (World Scientific, 2009).

${ }^{61}$ A. P. Lyubartsev and S. Marĕlja, Phys. Rev. E 65, 041202 (2002).

${ }^{62}$ A. Ben-Naim, Statistical Thermodynamics for Chemists and Biochemists (Plenum, New York, 1992).

${ }^{63}$ See supplementary material at http://dx.doi.org/10.1063/1.3693330 for the RDFs of the other ion-pairs considered in this work.

${ }^{64}$ R. A. Robinson and R. H. Stokes, Electrolyte Solutions, 2nd ed. (Dover, New York, 2002).

${ }^{65}$ B. Hess, J. Chem. Phys. 116, 209 (2002).

${ }^{66}$ K. D. Collins, Biophys. J. 72, 65 (1997).

${ }^{67}$ C. J. Fennell, A. Bizjak, V. Vlachy, and K. A. Dill, J. Phys. Chem. B 113, 6782 (2009).

${ }^{68}$ K. Fajans, J. Phys. Chem. 74, 3407 (1970).

${ }^{69}$ H. Coker, J. Phys. Chem. 80, 2084 (1976).

${ }^{70}$ R. R. Netz, Curr. Opin. Colloid Interface Sci. 9, 192 (2004). 